\title{
Treatment Patterns and Economic Burden by Lines of Therapy Among Patients with Advanced Hepatocellular Carcinoma Treated with Systemic Cancer Therapy
}

\author{
Machaon M. Bonafede ${ }^{1} \cdot$ Beata Korytowsky $^{2} \cdot$ Prianka Singh $^{2} \cdot$ Qian Cai $^{1} \cdot$ Katherine Cappell $^{1}$. \\ Krutika Jariwala-Parikh ${ }^{1} \cdot$ Bruce Sill $^{2} \cdot$ Neehar D. Parikh $^{3}$
}

Published online: 23 April 2019

(C) The Author(s) 2019

\begin{abstract}
Purpose This study examined clinical and economic outcomes among patients with advanced hepatocellular carcinoma (HCC) treated with systemic agents by line of therapy.

Methods Adults with $\geq 2$ medical claims for primary diagnosed HCC (from January 1, 2008, through September 30, 2015) and $\geq 1$ claim for systemic HCC-related therapy were identified in the IBM MarketScan ${ }^{\circledR}$ Research Databases. Continuous enrollment was required 6 months before and 1 month after diagnosis. Patients were categorized into first- (1L) and second-line (2L) treatment cohorts; those receiving sorafenib as $1 \mathrm{~L}$ were evaluated. Treatment patterns, healthcare resource utilization, costs, and survival during $1 \mathrm{~L}$ and $2 \mathrm{~L}$ therapy were measured. Survival was assessed for patients linked to the Social Security Administration Master Death File.

Results 1459 patients, 758 with death data, met the $1 \mathrm{~L}$ cohort criteria; 163 patients, 87 with death data, later received $2 \mathrm{~L}$ therapy. $77.1 \%$ had $1 \mathrm{~L}$ sorafenib, alone or in combination. Median $1 \mathrm{~L}$ treatment duration was 3.0 months; median survival time from start of $1 \mathrm{~L}$ to death or censor was 6.8 months. There was no predominant $2 \mathrm{~L}$ agent. Median $2 \mathrm{~L}$ treatment duration was 3.0 months; median survival time from start of $2 \mathrm{~L}$ was 9.3 months. Median total healthcare costs per patient per month were $\$ 13,297$ for $1 \mathrm{~L}$ (all), $\$ 13,471$ for $1 \mathrm{~L}$ (sorafenib), and $\$ 11,786$ for $2 \mathrm{~L}$.

Conclusions Findings confirm high 1-year mortality for advanced HCC, suggesting a high cost burden. While no 2L therapy was available during this analysis, recently approved $2 \mathrm{~L}$ agents have the potential to improve survival after sorafenib failure or intolerance.
\end{abstract}

Keywords Advanced hepatocellular carcinoma $\cdot$ Treatment patterns $\cdot$ Sorafenib $\cdot$ Cost burden $\cdot$ Line of therapy $\cdot$ Survival

\section{Introduction}

Hepatocellular carcinoma (HCC) is the most common primary liver cancer in the USA. The incidence of HCC tripled

Electronic supplementary material The online version of this article (https://doi.org/10.1007/s12029-019-00230-z) contains supplementary material, which is available to authorized users.

Machaon M. Bonafede

mbonafed@us.ibm.com

1 IBM Watson Health (formerly Truven Health Analytics Inc.), 75 Binney Street, Cambridge, MA 02142, USA

2 Bristol-Myers Squibb, Princeton, NJ, USA

3 Department of Internal Medicine, University of Michigan, Ann Arbor, MI, USA between 1975 and 2005 and has continued to increase, reaching an incidence of 6.7 cases per 100,000 persons in 2012 [1-4]. The leading risk factors for HCC are conditions that lead to liver cirrhosis, such as alcoholic liver disease, nonalcoholic steatohepatitis, or chronic infection with hepatitis B (HBV) or C virus (HCV) [1]. Patients diagnosed with early stage HCC who are eligible for surgical resection or transplantation can achieve 5 -year survival rates of over $60 \%$; therefore, there has been considerable focus on identification and monitoring of the at-risk population [2, 4]. However, for most patients who are diagnosed later with unresectable advanced HCC (aHCC) [3], 1-year survival is less than $40 \%$ and approved treatment options are limited $[5,6]$.

Sorafenib, an oral tyrosine kinase inhibitor with antiproliferative and antiangiogenic effects [7], was the first US Food and Drug Administration (FDA)-approved first-line (1L) therapy for the treatment of aHCC in 2007 [8]. Almost a decade 
later, in 2017, a second tyrosine kinase inhibitor, regorafenib, received FDA approval as a second-line (2L) therapy after disease progression on sorafenib $[9,10]$. Because of similar mechanisms of action, regorafenib is restricted to patients who are able to tolerate sorafenib [11]. Nivolumab, an antiprogrammed death-1 (PD-1) monoclonal antibody, was approved by the FDA in September 2017 as a 2L therapy for patients with aHCC after treatment with sorafenib [12]. More recently, lenvatinib, an oral tyrosine kinase inhibitor, was approved by the FDA for 1L therapy for patients with aHCC $[13,14]$. Cabozantinib (a tyrosine kinase inhibitor) and pembrolizumab (a PD-1 blocking antibody) are additional $2 \mathrm{~L}$ agents recently approved by the FDA for patients with aHCC previously treated with sorafenib $[15,16]$. Regorafenib, ramucirumab, cabozantinib, nivolumab, and pembrolizumab are also recommended by the National Comprehensive Cancer Network guidelines for patients who progress on or after sorafenib [17].

Using the Surveillance, Epidemiology, and End Results (SEER)-Medicare linked database in 2009, the aggregate healthcare and lost productivity costs in the USA due to HCC was estimated to be $\$ 454.9$ million annually, with $\$ 63.3$ million attributed to aHCC [18]. It should be noted that although the SEER-Medicare dataset is comprehensive, it only includes cost data on patients over the age of 65 years with Medicare coverage, limiting the generalizability; therefore, cost estimates in younger patients are likely to be imprecise [19]. Other studies that were based on healthcare costs of HCC in general and aHCC specifically were limited by small sample size or utilized data that predate the approval of sorafenib $[20,21]$.

Given the limited evidence on pharmacological treatment patterns and economic outcomes for aHCC, we evaluated the real-world evidence on systemic therapy use, healthcare resource utilization, associated costs, and mortality by lines of therapy in the USA.

\section{Methods}

\section{Study Design and Data Source}

This retrospective cohort study used de-identified patient-level administrative claims data to analyze demographics, duration of therapy, healthcare resource utilization, costs, and mortality for patients with $\mathrm{HCC}$ on $1 \mathrm{~L}$ and $2 \mathrm{~L}$ systemic cancer therapy. Healthcare resource utilization, costs, and patient characteristics were extracted from the IBM MarketScan ${ }^{\circledR}$ Commercial and Medicare Supplemental Databases for the period between July 1, 2007, and March 31, 2016. The Commercial Database contains the pharmacy and medical (inpatient and outpatient) claims of employees and their dependents, and the Medicare Supplemental Database contains the healthcare claims of individuals with Medicare supplemental insurance paid for by employers. Both databases provide information about resource utilization and associated costs for healthcare services performed in both inpatient and outpatient settings. The MarketScan Research Databases comprises approximately 30 million patients annually covered by a geographically diverse group of self-insured employers and private insurance plans across the United States. The MarketScan Research Databases were further linked to the Social Security Administration Master Death File to obtain patient death events.

Patient Selection and Study Cohorts Adult patients, aged $\geq 18$ years, who had at least two non-diagnostic medical claims (30-180 days apart) with a primary HCC diagnosis (International Classification of Diseases, Ninth Revision, Clinical Modification [ICD-9-CM] 155.0x) between January 1, 2008, and September 30, 2015, were identified. The date of the earliest medical claim for HCC diagnosis was defined as the diagnosis index date. Patients were required to have continuous medical and pharmacy coverage for at least 6 months before and 30 days after the diagnosis index date. Patients with a diagnosis of primary or secondary cancer (including HCC) or with a claim for a systemic cancer agent before the diagnosis index date as well as those with a diagnosis of cholangiocarcinoma (ICD-9-CM 155.1×) were excluded from the study.

1L cohort

Eligible patients were included in the $1 \mathrm{~L}$ cohort if they had at least one claim for an HCC-related systemic therapy, such as chemotherapy, targeted therapy, or immunotherapy, after the diagnosis index date. The $1 \mathrm{~L}$ index date was defined as the date of the first claim for the $1 \mathrm{~L}$ therapy. Patients were required to have continuous enrollment of 30 days after the $1 \mathrm{~L}$ index date. A full list of HCC-related systemic therapies used in patient identification and analysis can be found in Electronic Supplementary Material 1 . The subcohort of patients on $1 \mathrm{~L}$ sorafenib was also identified and analyzed.

2L cohort

Patients from the $1 \mathrm{~L}$ cohort were included in the $2 \mathrm{~L}$ cohort if they had at least one claim for a non-1L systemic agent after the $1 \mathrm{~L}$ index date. The $2 \mathrm{~L}$ index date was defined as the date of the first claim for $2 \mathrm{~L}$ therapy. To be included, patients had to have continuous enrollment from the diagnosis index date to 30 days after the $2 \mathrm{~L}$ index date. Any HCC-related systemic therapies initiated within 60 days of the $1 \mathrm{~L}$ and $2 \mathrm{~L}$ index date were considered part of $1 \mathrm{~L} / 2 \mathrm{~L}$ therapy. Some patients may have had more than one drug as a part of $1 \mathrm{~L} / 2 \mathrm{~L}$ therapy if they had initiated more than one drug within 60 days of $1 \mathrm{~L} / 2 \mathrm{~L}$ index date. Please see 'Outcome Measures' below for further detail on the definition of $1 \mathrm{~L}$ and $2 \mathrm{~L}$ therapy.

Patients on a $1 \mathrm{~L}$ or $2 \mathrm{~L}$ chemotherapy agent were excluded if they had an embolization (Current Procedural Terminology 
codes $37204,75894,36245,36246,36248,75896$, or 37243 ) within 30 days before or after the relevant index date. The intent of this criterion was to exclude patients who may have had an intratumoral infusion of chemotherapy treatment as part of transarterial chemoembolization and not as a systemic treatment.

\section{Baseline Characteristics}

Patient demographics, including age, sex, geographic region (US census division), urban or rural residency, and type of insurance were reported. Urban or rural residence classification was based on whether the primary subscriber's address was located within a Metropolitan Statistical Area. Recorded clinical characteristics included the Deyo-Charlson Comorbidity Index, an indicator of overall disease burden on the occurrence of at least one of 17 comorbid conditions identified using the ICD-9-CM coding manual. In addition to the Deyo-Charlson Comorbidity Index, general comorbid conditions (anxiety, cardiovascular disease, chronic obstructive pulmonary disease/asthma, depression, hypertension, osteoarthritis, and osteoporosis), liver-related conditions (nonalcoholic steatohepatitis, alcoholic liver disease, HBV status, and HCV status), and cirrhosis status were also assessed.

Demographic characteristics were reported on the cohortspecific index date ( $1 \mathrm{~L}$ or $2 \mathrm{~L}$ index date). Clinical characteristics were measured during the 6-month period before the cohort-specific index date.

\section{Outcome Measures}

The duration of therapy was assessed for $1 \mathrm{~L}$ and $2 \mathrm{~L}$, calculated from the $1 \mathrm{~L}$ and $2 \mathrm{~L}$ index dates to the earliest of the following: death, end of continuous enrollment, end of data (March 31, 2016), discontinuation, switch, or augmentation. Discontinuation was defined as a gap of $\geq 60$ days in medication supply, switching was defined as initiation of a new systemic cancer therapy without continued use of the index therapy $>60$ days after the $1 \mathrm{~L}$ or $2 \mathrm{~L}$ index date, and augmentation was defined as the initiation of a new systemic cancer therapy with continued use of the index therapy $>60$ days after the $1 \mathrm{~L}$ or $2 \mathrm{~L}$ index date. In addition to duration of therapy, time to initiation of $1 \mathrm{~L} / 2 \mathrm{~L}$ therapy, reason for terminating a line of therapy, and type of systemic drug(s) at $1 \mathrm{~L}$ and $2 \mathrm{~L}$ index were recorded.

All-cause healthcare resource utilization - including inpatient admissions, emergency room visits, and outpatient services such as physician office visits, laboratory tests, and radiology exams - was recorded for each cohort $1 \mathrm{~L}$ all, $1 \mathrm{~L}$ sorafenib, and $2 \mathrm{~L}$ all), for duration of therapy. For patients in the $1 \mathrm{~L}$ sorafenib cohort, the time to first inpatient admission and the treatment quartile of each inpatient admission were recorded to determine the timing of inpatient stays during the course of treatment. Treatment quartiles were determined on a per-patient basis as one-quarter of a patient's duration of therapy.

All-cause total healthcare costs, including medical services costs (inpatient admissions, emergency room visits, and outpatient services) and outpatient pharmacy costs over the duration of therapy, were reported for the $1 \mathrm{~L}$ and $2 \mathrm{~L}$ cohorts. Total costs were defined as the sum of health plan and patient-paid costs incurred from fully adjudicated medical and pharmacy claims. All costs were reported as per patient per month and adjusted to 2015 US dollars using the medical care component of the Consumer Price Index [22]. End of follow-up for healthcare utilization and cost outcomes was the earliest instance of inpatient death or Social Security Administration death, end of continuous enrollment, or end of study (i.e., March 31, 2016).

To evaluate survival outcomes, the analysis was limited to the subset of patients in the commercial and Medicare databases that could be linked to the Social Security Administration death data. Among the subset of linked patients, we assessed whether they experienced death or not. Date of death was determined based on the death record from the linked Social Security Administration Master Death File. Survival time was calculated as the time from the $1 \mathrm{~L}$ and $2 \mathrm{~L}$ index date to the death date (if the patient died) or censor date (end of continuous enrollment or study period), whichever occurred first.

Statistical Analysis For all study variables, mean, standard deviation, and median were reported for all continuous variables, and frequencies and percentages were reported for categorical variables. All data analyses were conducted using SAS version 9.4 (SAS Inc., Cary, NC, USA). Data availability: Bristol-Myers Squibb policy on data sharing may be found at https://www.bms.com/researchers-and-partners/ independent-research/data-sharing-request-process.html.

\section{Results}

\section{Demographic and Clinical Characteristics}

A total of 1459 patients met the inclusion criteria for the $1 \mathrm{~L}$ cohort. Of these patients, $163(11.2 \%)$ met the criteria for inclusion in the $2 \mathrm{~L}$ cohort (Fig. 1). The mean age of patients in the $1 \mathrm{~L}$ cohort was $61.7 \pm 10.1$ years and the majority were males $(78.1 \%)$. Most patients $(77.1 \%)$ were treated with $1 \mathrm{~L}$ sorafenib; the mean age of this subcohort was $62.0 \pm$ 10.1 years and $81.4 \%$ were male. The mean age of the $2 \mathrm{~L}$ cohort was $61.0 \pm 9.1$ years and $74.2 \%$ were males (Table 1). Over $40 \%$ of patients from all cohorts were from the US South Census region and more than $85 \%$ were urban residents. The majority had health insurance coverage through 
Fig. 1 Patient selection. ICD-9$C M$ International Classification of Diseases, ninth revision, clinical modification, SSA DMF Social Security Administration Death Master File. Superscript a, the look-back period began on January 1, 2005 (or start of database enrollment, whichever is later), and ended on the day before the index date; superscript $\mathrm{b}$, for list see Electronic Supplementary Material 1; superscript c, excludes patients with $1 \mathrm{~L}$ chemotherapy who had an embolization within \pm 30 days of $1 \mathrm{~L}$ index date

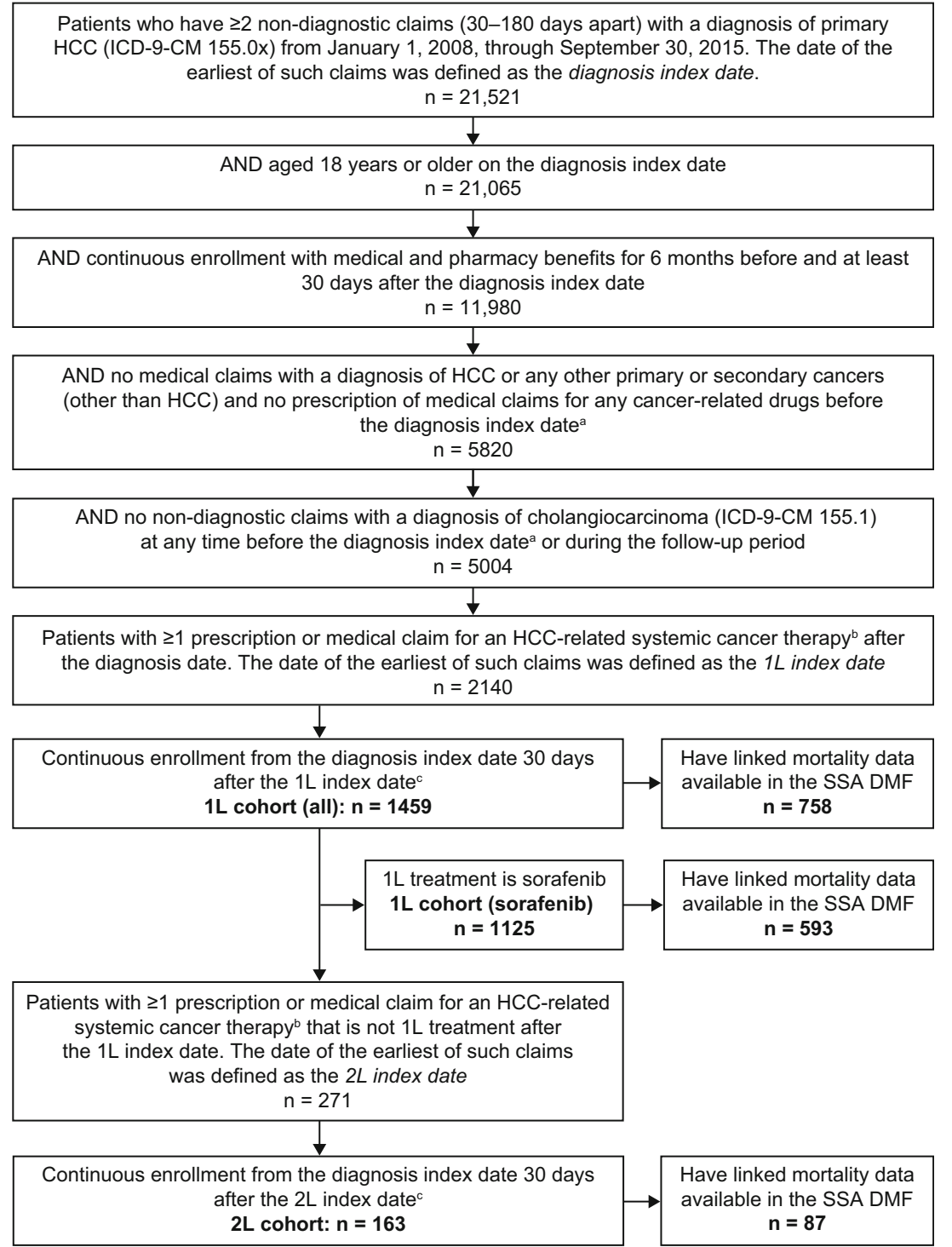

exclusive provider organizations or preferred provider organizations (Table 1).

The mean baseline Deyo-Charlson Comorbidity Index of patients in the 1L (all), 1L (sorafenib), and 2L cohorts was 5.7 $\pm 3.1,5.5 \pm 3.1$, and $6.3 \pm 3.3$, respectively. The top three comorbid conditions at baseline were hypertension (1L-all, $41.7 \%$; 1L-sorafenib, 41.0\%; 2L, 36.2\%), diabetes (1L-all, $33.7 \%$; 1L-sorafenib, 35.3\%; 2L, 27.0\%), and cardiovascular disease (1L-all, 33.5\%; 1L-sorafenib, 33.3\%; 2L, 28.2\%). The frequency of cirrhosis was much higher than that of alcoholic liver disease in the pre-index period for all three cohorts: $44.3 \%$ versus $11.5 \%$ in the $1 \mathrm{~L}$ (all), $48.3 \%$ versus $12.5 \%$ in $1 \mathrm{~L}$ (sorafenib) subcohort, and $27 \%$ versus $4.9 \%$ in the $2 \mathrm{~L}$ cohort. Among the 1L (all) cohort, $38 \%$ of patients had HCV only, $5.8 \%$ had HBV only, and $2.4 \%$ had both HCV and $\mathrm{HBV}$; a similar distribution was found in the $1 \mathrm{~L}$ (sorafenib) subcohort and the 2L cohort.
Treatment Patterns The treatment patterns for the $1 \mathrm{~L}$ and $2 \mathrm{~L}$ cohorts are shown in Table 2. In brief, the mean (median) time from diagnosis index date to $1 \mathrm{~L}$ index date was $5.24 \pm$ 8.93 (1.63) months. For patients who progressed to $2 \mathrm{~L}$ treatment, the mean (median) time from the end of $1 \mathrm{~L}$ therapy to the $2 \mathrm{~L}$ index date was $3.06 \pm 5.39$ (1.07) months. Sorafenib monotherapy was the most commonly prescribed $1 \mathrm{~L}$ therapy and the mean time to treatment initiation for this subcohort was $4.17 \pm 7.02$ months. For $2 \mathrm{~L}$ therapies, the most common choices were systemic chemotherapy $(n=$ $80 ; 49.1 \%)$ and targeted therapy other than sorafenib $(n=$ $70 ; 42.9 \%)$. Across the patient population, a total of 68 different $1 \mathrm{~L}$ and 44 different $2 \mathrm{~L}$ treatment regimens, including combinations, were identified. The top five regimens used by patients in the $1 \mathrm{~L}$ cohort included sorafenib (74\%); sirolimus (6\%); everolimus (2\%); gemcitabine (2\%); and doxorubicin and sorafenib combination $(2 \%)$ 
Table 1 Demographic and clinical characteristics

\begin{tabular}{|c|c|c|c|c|c|c|}
\hline & \multirow{2}{*}{\multicolumn{2}{|c|}{$\begin{array}{l}\text { First-line cohort } \\
\text { (all) }\end{array}$}} & \multirow{2}{*}{\multicolumn{2}{|c|}{$\begin{array}{l}\begin{array}{l}\text { First-line cohort } \\
\text { (sorafenib) }\end{array} \\
N=1125\end{array}$}} & \multirow{2}{*}{\multicolumn{2}{|c|}{$\begin{array}{l}\begin{array}{l}\text { Second-line } \\
\text { cohort (all) }\end{array} \\
N=163\end{array}$}} \\
\hline & & & & & & \\
\hline \multicolumn{7}{|l|}{ Demographics } \\
\hline Age (mean, SD), years & 61.7 & 10.1 & 62.0 & 10.1 & 61.0 & 9.1 \\
\hline Male $(N, \%)$ & 1140 & $78.1 \%$ & 916 & $81.4 \%$ & 121 & $74.2 \%$ \\
\hline \multicolumn{7}{|l|}{ Geographic region $(N, \%)$} \\
\hline Northeast & 260 & $17.8 \%$ & 204 & $18.1 \%$ & 36 & $22.1 \%$ \\
\hline North Central & 288 & $19.7 \%$ & 211 & $18.8 \%$ & 31 & $19.0 \%$ \\
\hline South & 593 & $40.6 \%$ & 454 & $40.4 \%$ & 69 & $42.3 \%$ \\
\hline West & 290 & $19.9 \%$ & 236 & $21.0 \%$ & 25 & $15.3 \%$ \\
\hline Unknown & 28 & $1.9 \%$ & 20 & $1.8 \%$ & 2 & $1.2 \%$ \\
\hline \multicolumn{7}{|l|}{ Insurance plan type $(N, \%)$} \\
\hline Comprehensive/indemnity & 218 & $14.9 \%$ & 180 & $16.0 \%$ & 23 & $14.1 \%$ \\
\hline $\mathrm{EPO} / \mathrm{PPO}$ & 774 & $53.1 \%$ & 587 & $52.2 \%$ & 85 & $52.2 \%$ \\
\hline POS/POS with capitation & 85 & $5.8 \%$ & 71 & $6.3 \%$ & 11 & $6.8 \%$ \\
\hline HMO & 252 & $17.3 \%$ & 198 & $17.6 \%$ & 25 & $15.3 \%$ \\
\hline CDHP/HDHP & 73 & $5.0 \%$ & 50 & $4.4 \%$ & 13 & $8.0 \%$ \\
\hline Unknown & 57 & $3.9 \%$ & 39 & $3.5 \%$ & 6 & $3.7 \%$ \\
\hline \multicolumn{7}{|l|}{ Rural residence indicator $(N, \%)$} \\
\hline Urban & 1250 & $85.7 \%$ & 976 & $86.8 \%$ & 140 & $85.9 \%$ \\
\hline Rural & 182 & $12.5 \%$ & 129 & $11.5 \%$ & 21 & $12.9 \%$ \\
\hline Unknown & 27 & $1.9 \%$ & 20 & $1.8 \%$ & 2 & $1.2 \%$ \\
\hline \multicolumn{7}{|l|}{ Clinical characteristics } \\
\hline Deyo-Charlson Comorbidity Index (mean, SD) & 5.7 & 3.1 & 5.5 & 3.1 & 6.3 & 3.3 \\
\hline \multicolumn{7}{|l|}{ Comorbid conditions $(N, \%)$} \\
\hline Anxiety & 59 & $4.0 \%$ & 44 & $3.9 \%$ & 8 & $4.9 \%$ \\
\hline Cardiovascular disease & 488 & $33.5 \%$ & 375 & $33.3 \%$ & 46 & $28.2 \%$ \\
\hline Chronic obstructive pulmonary disease/asthma & 141 & $9.7 \%$ & 115 & $10.2 \%$ & 13 & $8.0 \%$ \\
\hline Depression & 72 & $4.9 \%$ & 52 & $4.6 \%$ & 14 & $8.6 \%$ \\
\hline Diabetes & 491 & $33.7 \%$ & 397 & $35.3 \%$ & 44 & $27.0 \%$ \\
\hline Hypertension & 608 & $41.7 \%$ & 461 & $41.0 \%$ & 59 & $36.2 \%$ \\
\hline Osteoarthritis & 89 & $6.1 \%$ & 72 & $6.4 \%$ & 5 & $3.1 \%$ \\
\hline Osteoporosis & 11 & $0.8 \%$ & 5 & $0.4 \%$ & 1 & $0.6 \%$ \\
\hline \multicolumn{7}{|l|}{ Liver-related comorbidities $(N, \%)$} \\
\hline Non-alcoholic steatohepatitis & 76 & $5.2 \%$ & 59 & $5.2 \%$ & 7 & $4.3 \%$ \\
\hline Alcoholic liver disease & 167 & $11.5 \%$ & 141 & $12.5 \%$ & 8 & $4.9 \%$ \\
\hline Hepatitis B virus only & 84 & $5.8 \%$ & 74 & $6.6 \%$ & 3 & $1.8 \%$ \\
\hline Hepatitis $\mathrm{C}$ virus only & 554 & $38.0 \%$ & 458 & $40.7 \%$ & 58 & $35.6 \%$ \\
\hline Hepatitis B and C virus & 35 & $2.4 \%$ & 26 & $2.3 \%$ & 3 & $1.8 \%$ \\
\hline Cirrhosis status & 646 & $44.3 \%$ & 543 & $48.3 \%$ & 44 & $27.0 \%$ \\
\hline
\end{tabular}

$C D H P$, consumer-driven health plan; $E P O$, exclusive provider organization; $H D H P$, high-deductible health plan; $H M O$, health maintenance organization; $P O S$, point of service; $P P O$, preferred provider organization; $S D$, standard deviation (see Electronic Supplementary Material 2). The top five regimens used by patients in the $2 \mathrm{~L}$ cohort included sirolimus (17\%); fluorouracil (8\%); sorafenib (7\%); capecitabine (7\%); everolimus $(6 \%)$; and gemcitabine $(6 \%)$ (see Electronic Supplementary Material 3).
The mean duration of therapy was $5.06 \pm 5.98$ months with a median of 3.03 months in the 1L (all) cohort, $4.90 \pm$ 5.63 months with a median of 3.00 months in the $1 \mathrm{~L}$ (sorafenib) subcohort, and $5.13 \pm 7.29$ months with a median of 3.03 months in the $2 \mathrm{~L}$ cohort. 
Table 2 Treatment patterns

\begin{tabular}{|c|c|c|c|c|c|c|}
\hline & \multicolumn{2}{|c|}{$\begin{array}{l}\text { First-line cohort } \\
\text { (all) }\end{array}$} & \multicolumn{2}{|c|}{$\begin{array}{l}\text { First-line cohort } \\
\text { (sorafenib) }\end{array}$} & \multicolumn{2}{|c|}{$\begin{array}{l}\text { Second-line } \\
\text { cohort (all) }\end{array}$} \\
\hline & $N=1459$ & & $N=1125$ & & $N=163$ & \\
\hline Months to treatment start (mean, SD) & 5.24 & 8.93 & 4.17 & 7.02 & 3.06 & 5.39 \\
\hline Months to start (median) & 1.63 & & 1.43 & & 1.07 & \\
\hline \multicolumn{7}{|l|}{ Index therapy ${ }^{\mathrm{a}, \mathrm{b}}(\mathrm{N}, \%)$} \\
\hline Systemic chemotherapy & 181 & $12.4 \%$ & 1 & $0.1 \%$ & 80 & $49.1 \%$ \\
\hline Targeted therapies without sorafenib & 165 & $11.3 \%$ & 0 & $0.0 \%$ & 70 & $42.9 \%$ \\
\hline Immunotherapy & 1 & $0.1 \%$ & 0 & $0.0 \%$ & 4 & $2.5 \%$ \\
\hline Sorafenib & 1125 & $77.1 \%$ & 1125 & $100.0 \%$ & 15 & $9.2 \%$ \\
\hline \multicolumn{7}{|l|}{ Reason for termination $(N, \%)$} \\
\hline Discontinuation & 609 & $41.7 \%$ & 451 & $40.1 \%$ & 70 & $42.9 \%$ \\
\hline Switching & 81 & $5.6 \%$ & 54 & $4.8 \%$ & 17 & $10.4 \%$ \\
\hline Augmentation & 29 & $2.0 \%$ & 15 & $1.3 \%$ & 8 & $4.9 \%$ \\
\hline Death & 190 & $13.0 \%$ & 169 & $15.0 \%$ & 12 & $7.4 \%$ \\
\hline End of enrollment & 515 & $35.3 \%$ & 421 & $37.4 \%$ & 44 & $27.0 \%$ \\
\hline End of study & 35 & $2.4 \%$ & 15 & $1.3 \%$ & 12 & $7.4 \%$ \\
\hline Duration of therapy, months (mean, SD) & 5.06 & 5.98 & 4.90 & 5.63 & 5.13 & 7.29 \\
\hline Duration of therapy, months (median) & 3.03 & & 3.00 & & 3.03 & \\
\hline Duration of 2L sorafenib, months (mean, SD) & & & & & 6.82 & 4.06 \\
\hline Duration of $2 \mathrm{~L}$ sorafenib, months (median) & & & & & 7.47 & \\
\hline
\end{tabular}

$2 L$, second line; $S D$, standard deviation

${ }^{\text {a }}$ Patients can receive more than one type of medication on the index date

${ }^{\mathrm{b}}$ See Online Resource Material 1 for a full list of therapies

Healthcare Resource Utilization and Costs During 1L therapy, $42.9 \%$ of patients in the $1 \mathrm{~L}$ cohort had at least one inpatient admission, and $38.6 \%$ had at least one emergency room visit (Table 3). Of the 499 patients in the $1 \mathrm{~L}$ sorafenib subcohort with at least one inpatient admission, $53 \%$ had their first inpatient admission during the first half of their therapy (Fig. 2). The number of inpatient readmissions increased steadily through the fourth treatment quartile.

Mean all-cause per-patient per-month total healthcare costs for $1 \mathrm{~L}$ therapy were $\$ 18,381 \pm \$ 19,633$ and median costs were $\$ 13,297$. In the $1 \mathrm{~L}$ sorafenib subcohort, $40.1 \%$ had at least one emergency room visit (Table 3). Mean all-cause per-patient per-month total healthcare costs for this subcohort were $\$ 18,559 \pm \$ 18,012$ and median costs were $\$ 13,471$.

During 2L therapy, $33.7 \%$ of patients had at least one inpatient admission, and $20.3 \%$ had at least one emergency room visit (Table 3). Mean all-cause per-patient per-month total healthcare costs for $2 \mathrm{~L}$ therapy were $\$ 19,559 \pm \$ 30,065$ and median costs were $\$ 11,786$. Medical costs represented $65 \%$ of $1 \mathrm{~L}$ (all) healthcare costs, $59 \%$ of $1 \mathrm{~L}$ (sorafenib) healthcare costs, and $82 \%$ of $2 \mathrm{~L}$ healthcare costs. Additional detail on healthcare resource utilization and costs for all cohorts can be found in Table 3 .

\section{Survival Outcomes}

More than half of the study patients could be linked to the Social Security Administration Master Death File $(n=758$ for 1L [all] cohort; $n=593$ for 1L [sorafenib] subcohort; $n=$ 87 for $2 \mathrm{~L}$ cohort), enabling the assessment of survival outcomes. Of them, more than half died during the follow-up period $(54.0 \%$ of the $1 \mathrm{~L}$ [all], $58.2 \%$ of the $1 \mathrm{~L}$ [sorafenib], and $52.9 \%$ of the $2 \mathrm{~L}$ cohort; Table 4$)$. Median survival time, from cohort $1 \mathrm{~L}$ and $2 \mathrm{~L}$ index date to death or censor, was 6.8 months for $1 \mathrm{~L}$ (all) patients, 6.0 months for $1 \mathrm{~L}$ (sorafenib) patients, and 9.3 months for $2 \mathrm{~L}$ patients. Oneyear survival was $32.6 \%$ for $1 \mathrm{~L}$ (all) therapy, $28.5 \%$ for $1 \mathrm{~L}$ (sorafenib), and $34.5 \%$ for $2 \mathrm{~L}$ therapy. Survival rates at 3 years were $6.6 \%$ for $1 \mathrm{~L}$ (all) therapy, $5.4 \%$ for $1 \mathrm{~L}$ sorafenib, and $5.8 \%$ for $2 \mathrm{~L}$ therapy.

\section{Discussion}

This retrospective claims study is the first real-world analysis that presents comprehensive data on treatment patterns, healthcare resource utilization, healthcare costs, and survival outcomes by line of therapy in patients with aHCC who 
Table 3 All-cause healthcare resource utilization and costs

\begin{tabular}{|c|c|c|c|c|c|c|c|c|c|}
\hline & \multicolumn{3}{|c|}{ First-line cohort (all) } & \multicolumn{3}{|c|}{ First-line cohort (sorafenib) } & \multicolumn{3}{|c|}{ Second-line cohort (all) } \\
\hline & \multicolumn{3}{|c|}{$N=1459$} & \multicolumn{3}{|l|}{$N=1125$} & \multicolumn{3}{|l|}{$N=163$} \\
\hline & $N /$ mean & $\% / \mathrm{SD}$ & Median & $N /$ mean & $\% / \mathrm{SD}$ & Median & $N /$ mean & $\% / \mathrm{SD}$ & Median \\
\hline \multicolumn{10}{|l|}{ Medical services } \\
\hline \multicolumn{10}{|l|}{ IP admissions } \\
\hline Patients with any IP admission $(N, \%)$ & 626 & $42.9 \%$ & & 499 & $44.4 \%$ & & 55 & $33.7 \%$ & \\
\hline No. of IP admissions, PPPM $^{\mathrm{a}}$ (mean, SD) & 0.2 & 0.4 & & 0.2 & 0.4 & & 0.2 & 0.4 & \\
\hline IP costs, PPPM (mean, SD, median) & $\$ 5605$ & $\$ 14,887$ & $\$ 0$ & $\$ 5705$ & $\$ 12,561$ & $\$ 0$ & $\$ 4635$ & $\$ 23,174$ & $\$ 0$ \\
\hline \multicolumn{10}{|l|}{ ER visits } \\
\hline Patients with any ER visit $(N, \%)$ & 563 & $38.6 \%$ & & 451 & $40.1 \%$ & & 33 & $20.3 \%$ & \\
\hline No. of ER visits, PPPM (mean, SD) & 0.3 & 0.7 & & 0.4 & 0.8 & & 0.3 & 1.6 & \\
\hline ER costs, PPPM (mean, SD, median) & $\$ 246$ & $\$ 1727$ & $\$ 0$ & $\$ 206$ & $\$ 808$ & $\$ 0$ & $\$ 121$ & $\$ 481$ & $\$ 0$ \\
\hline \multicolumn{10}{|l|}{ OP services } \\
\hline Total OP costs, PPPM (mean, SD, median) & $\$ 6118$ & $\$ 11,517$ & $\$ 2723$ & $\$ 5067$ & $\$ 11,681$ & $\$ 2144$ & $\$ 11,194$ & $\$ 18,156$ & $\$ 5761$ \\
\hline \multicolumn{10}{|l|}{ Physician office visits } \\
\hline No. of office visits, PPPM (mean, SD) & 2.2 & 1.6 & & 2.1 & 1.5 & & 2.4 & 2.1 & \\
\hline Office visit costs, PPPM (mean, SD, median) & $\$ 303$ & $\$ 506$ & $\$ 217$ & $\$ 305$ & $\$ 533$ & $\$ 215$ & $\$ 355$ & $\$ 840$ & $\$ 210$ \\
\hline \multicolumn{10}{|l|}{ Laboratory services } \\
\hline No. of laboratory services, PPPM (mean, SD) & 2.2 & 2.0 & & 1.9 & 1.7 & & 2.8 & 2.4 & \\
\hline Laboratory costs, PPPM (mean, SD, median) & $\$ 401$ & $\$ 749$ & $\$ 138$ & $\$ 328$ & $\$ 645$ & $\$ 112$ & $\$ 419$ & $\$ 639$ & $\$ 173$ \\
\hline \multicolumn{10}{|l|}{ Radiology services } \\
\hline No. of radiology services, PPPM (mean, SD) & 1.5 & 2.4 & & 1.4 & 2.1 & & 2.0 & 3.9 & \\
\hline Radiology costs, PPPM (mean, SD, median) & $\$ 1187$ & $\$ 3414$ & $\$ 289$ & $\$ 1134$ & $\$ 3512$ & $\$ 240$ & $\$ 2187$ & $\$ 8981$ & $\$ 414$ \\
\hline \multicolumn{10}{|l|}{ Other OP services } \\
\hline No. of other OP services, PPPM (mean, SD) & 4.3 & 5.1 & & 3.6 & 3.9 & & 7.2 & 8.8 & \\
\hline Other OP service costs, PPPM (mean, SD, median) & $\$ 4227$ & $\$ 10,195$ & $\$ 1286$ & $\$ 3300$ & $\$ 10,326$ & $\$ 900$ & $\$ 8232$ & $\$ 14,631$ & $\$ 4037$ \\
\hline \multicolumn{10}{|l|}{ OP pharmacy } \\
\hline No. of OP pharmacy claims, PPPM (mean, SD) & 5.3 & 5.5 & & 5.0 & 3.0 & & 5.2 & 3.4 & \\
\hline OP pharmacy costs, PPPM (mean, SD, median) & $\$ 6413$ & $\$ 4728$ & $\$ 6245$ & $\$ 7581$ & $\$ 4006$ & $\$ 7514$ & $\$ 3609$ & $\$ 5252$ & $\$ 1271$ \\
\hline Total medical costs ${ }^{\mathrm{b}}$, PPPM (mean, SD, median) & $\$ 11,968$ & $\$ 19,130$ & $\$ 5879$ & $\$ 10,978$ & $\$ 17,438$ & $\$ 4973$ & $\$ 15,950$ & $\$ 30,179$ & $\$ 8142$ \\
\hline Total healthcare costs ${ }^{\mathrm{c}}, \mathrm{PPPM}$ (mean, SD, median) & $\$ 18,381$ & $\$ 19,633$ & $\$ 13,297$ & $\$ 18,559$ & $\$ 18,012$ & $\$ 13,471$ & $\$ 19,559$ & $\$ 30,065$ & $\$ 11,786$ \\
\hline
\end{tabular}

$E R$, emergency room; $I P$, inpatient; $O P$, outpatient; $P P P M$, per-patient-per-month; $S D$, standard deviation

${ }^{a}$ Counts and expenditures are reported PPPM due to the variable follow-up period

${ }^{\mathrm{b}}$ Medical costs include IP admission costs, ER visit costs, and OP services costs

${ }^{\mathrm{c}}$ Healthcare costs include medical costs and OP pharmacy costs

received systemic cancer therapy. Our findings showed that the overall survival outcomes were poor in both lines of therapy with high healthcare resource utilization and economic burden in both the $1 \mathrm{~L}$ and $2 \mathrm{~L}$ setting and in the sorafenib $1 \mathrm{~L}$ subcohort; only a low percentage of patients progressed to $2 \mathrm{~L}$ due to the burden of HCC and the lack of standardized treatment options. Sorafenib has been the standard of care in $1 \mathrm{~L}$ aHCC since its approval in 2005. However, there remains an unmet need for $2 \mathrm{~L}$ and $1 \mathrm{~L}$ alternatives to sorafenib that improve outcomes with fewer side effects while maintaining quality of life and cost savings.
In this high-cost population, medical expensesconsisting of inpatient hospitalization and outpatient services costs-exceeded $\$ 10,000(\$ 4500)$ per patient per month for both the $1 \mathrm{~L}$ and $2 \mathrm{~L}$ cohorts and in the $1 \mathrm{~L}$ sorafenib subcohort. In particular, the strong positive skew of cost data was driven by high rates of inpatient admissions; therefore, medications and management strategies that are both effective and minimize hospitalizations due to adverse events or disease-related complications are needed to maximize value [20]. Our results on the cost of systemic therapy for aHCC are consistent with previous 


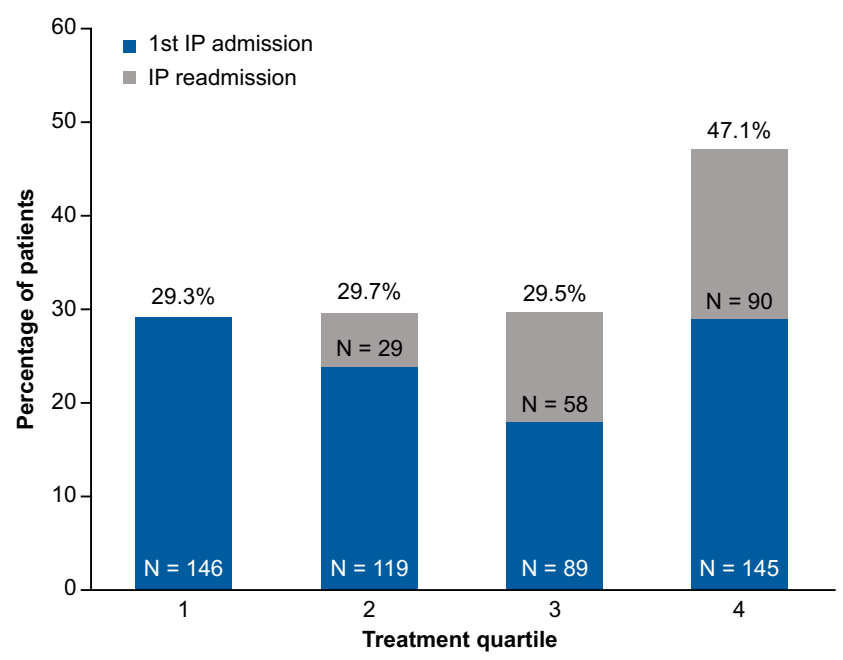

Fig. 2 Percentage of first-line sorafenib patients with at least one IP admission $(N=499)$ whose IP admission occurred within each treatment quartile. $I P$ inpatient

reports in which the estimated monthly healthcare costs of patients receiving $1 \mathrm{~L}$ sorafenib have ranged from $\$ 6000$ to over $\$ 16,000[20,23]$.

Given the trend towards value-based care [24], there is a need to have therapies evaluated for cost-effectiveness, a measure that incorporates both economic and clinical outcomes of the drug [25]. Recently, the tyrosine kinase inhibitor lenvatinib was approved for $1 \mathrm{~L}$ treatment of aHCC $[13,14]$, while the multikinase inhibitors regorafenib and cabozantinib and the immuno-oncology drugs nivolumab and pembrolizumab were approved as $2 \mathrm{~L}$ therapies $[9$, $10,12,26]$.
As the treatment landscape of aHCC shifts with the entry of newer agents, cancer-specific patient-reported outcome measures will be essential to capture differences in patient quality of life in cases where standard outcome metrics demonstrate equivalency between treatments [24, 27, 28]. For example, a recent review of patient-reported outcomes associated with nivolumab treatment of advanced solid cancers reported that, in addition to clinical benefits, nivolumab was associated with stabilization or improvement of patient quality of life [28]. This is in contrast to chemotherapy agents and targeted therapies that are generally associated with deterioration in quality of life.

\section{Limitations}

The limitations of this study are similar to those seen in other claims-based observational studies. First, this study was restricted to those individuals with commercial health coverage or private Medicare supplemental coverage; therefore, results may not be generalizable to patients with other insurance types or without health insurance coverage. This population is younger than the general aHCC population and may overestimate the efficacy and duration of treatment and healthrelated outcomes. Second, claim data are collected for administrative purposes; therefore, the data are subject to coding limitations and data entry errors, lack indicators of clinical status such as Eastern Cooperative Oncology Group scores or cancer staging, and only reflect direct healthcare costs via the paid amounts of adjudicated claims to individual hospitals and providers. Third, death data were only available for the subset of individuals who could be linked to the Social Security Administration Master Death File. Fourth, patients
Table 4 Survival during the follow-up period

\begin{tabular}{|c|c|c|c|c|c|c|}
\hline \multirow[b]{3}{*}{$\operatorname{Died}(N, \%)$} & \multirow{2}{*}{\multicolumn{2}{|c|}{$\begin{array}{l}\text { First-line cohort } \\
\text { (all) } \\
N=758\end{array}$}} & \multirow{2}{*}{\multicolumn{2}{|c|}{$\begin{array}{l}\begin{array}{l}\text { First-line cohort } \\
\text { (sorafenib) }\end{array} \\
N=593\end{array}$}} & \multirow{2}{*}{\multicolumn{2}{|c|}{$\begin{array}{l}\text { Second-line cohort } \\
\text { (all) } \\
N=87\end{array}$}} \\
\hline & & & & & & \\
\hline & 409 & $54.0 \%$ & 345 & $58.2 \%$ & 46 & $52.9 \%$ \\
\hline Survival time, months ${ }^{\mathrm{a}}$ (mean, SD) & 12.28 & 14.34 & 11.09 & 13.13 & 13.78 & 14.14 \\
\hline Survival time, months ${ }^{\mathrm{a}}$ (median) & 6.83 & & 5.97 & & 9.33 & \\
\hline \multicolumn{7}{|l|}{ Patients who survived $\mathrm{d}^{\mathrm{b}}(N, \%)$} \\
\hline$<1$ year & 511 & $67.4 \%$ & 424 & $71.5 \%$ & 57 & $65.5 \%$ \\
\hline$\geq 1$ & 247 & $32.6 \%$ & 169 & $28.5 \%$ & 30 & $34.5 \%$ \\
\hline$\geq 2$ & 94 & $12.4 \%$ & 66 & $11.1 \%$ & 14 & $16.1 \%$ \\
\hline$\geq 3$ & 50 & $6.6 \%$ & 32 & $5.4 \%$ & 5 & $5.7 \%$ \\
\hline$\geq 4$ & 30 & $4.0 \%$ & 18 & $3.0 \%$ & 4 & $4.6 \%$ \\
\hline
\end{tabular}


with aHCC who did not receive systemic cancer therapy, such as those who received only palliative care, were not evaluated in this study, and misclassification bias due to the implementation of line of therapy algorithms may occur in this study. Lastly, the study was completed before the approval of current $2 \mathrm{~L}$ therapies for aHCC, so the treatment landscape may look different in future analyses. However, given the recentness of this evolution in care, this study provides a baseline for survival, healthcare utilization and cost prior to the approval of these new agents.

\section{Conclusions}

In this real-world claims analysis of patients with aHCC, most patients received sorafenib as $1 \mathrm{~L}$ treatment; however, $23 \%$ of patients were treated with non-approved $1 \mathrm{~L}$ therapies and there were no apparent trends in the selection of $2 \mathrm{~L}$ therapies. Healthcare costs were substantial, primarily driven by pharmacy costs for the $1 \mathrm{~L}$ cohort and by outpatient services for the $2 \mathrm{~L}$ cohort. The high 1-year mortality rate and economic burden associated with aHCC underscores the continued need for more effective pharmacologic treatments for this patient population.

Acknowledgments Medical writing services were provided by Jessamine P. Winer-Jones, PhD, of IBM Watson Health (formerly Truven Health Analytics), and editorial assistance was provided by PAREXEL International. These services were paid for by Bristol-Myers Squibb.

Funding This study was funded by Bristol-Myers Squibb.

\section{Compliance with Ethical Standards}

Conflict of Interest Beata Korytowsky, Prianka Singh, and Bruce Sill are employed by Bristol-Myers Squibb. Machaon M. Bonafede, Katherine Cappell, and Krutika Jariwala-Parikh are employed by IBM Watson Health, which received a research contract to conduct this analysis with and on behalf of Bristol-Myers Squibb. Qian Cai was employed by IBM Watson Health at the time the study was conducted and is currently employed by the Janssen Pharmaceutical Companies of Johnson \& Johnson. Neehar D. Parikh is a paid consultant of Bristol-Myers Squibb. This research was presented in part at the 2017 AASLD Liver Meeting in San Francisco, CA, USA, and the 2018 ASCO Gastrointestinal Cancer Symposium in San Francisco, CA, USA.

Ethical Approval and Informed Consent All procedures performed in studies involving human participants were in accordance with the ethical standards of the institutional and/or national research committee and with the 1964 Helsinki declaration and its later amendments or comparable ethical standards. This article does not contain any studies with animals performed by any of the authors. All study data were accessed with protocols compliant with US patient confidentiality requirements, including the Health Insurance Portability and Accountability Act of 1996 regulations (HIPAA). As all databases used in the study are fully deidentified and compliant with the HIPAA, this study was exempted from Institutional Review Board approval.
Open Access This article is distributed under the terms of the Creative Commons Attribution 4.0 International License (http:// creativecommons.org/licenses/by/4.0/), which permits unrestricted use, distribution, and reproduction in any medium, provided you give appropriate credit to the original author(s) and the source, provide a link to the Creative Commons license, and indicate if changes were made.

\section{References}

1. El-Serag HB, Kanwal F. Epidemiology of hepatocellular carcinoma in the United States: where are we? Where do we go? Hepatology. 2014;60(5):1767-75.

2. Heimbach JK, Kulik LM, Finn RS, Sirlin CB, Abecassis MM, Roberts LR, et al. AASLD guidelines for the treatment of hepatocellular carcinoma. Hepatology. 2018;67(1):358-80.

3. Park JW, Chen M, Colombo M, Roberts LR, Schwartz M, Chen PJ, et al. Global patterns of hepatocellular carcinoma management from diagnosis to death: the BRIDGE study. Liver Int. 2015;35(9):215566.

4. Singal AG, Yopp A, Skinner CS, Packer M, Lee WM, Tiro JA. Utilization of hepatocellular carcinoma surveillance among American patients: a systematic review. J Gen Intern Med. 2012;27(7):861-7.

5. Morise Z, Kawabe N, Tomishige H, Nagata H, Kawase J, Arakawa $\mathrm{S}$, et al. Recent advances in the surgical treatment of hepatocellular carcinoma. World J Gastroenterol. 2014;20(39):14381-92.

6. Llovet JM, Bustamante J, Castells A, Vilana R, Ayuso Mdel C, Sala $\mathrm{M}$, et al. Natural history of untreated nonsurgical hepatocellular carcinoma: rationale for the design and evaluation of therapeutic trials. Hepatology. 1999;29(1):62-7.

7. Wilhelm SM, Adnane L, Newell P, Villanueva A, Llovet JM, Lynch M. Preclinical overview of sorafenib, a multikinase inhibitor that targets both Raf and VEGF and PDGF receptor tyrosine kinase signaling. Mol Cancer Ther. 2008;7(10):3129-40.

8. Llovet JM, Ricci S, Mazzaferro V, Hilgard P, Gane E, Blanc JF, et al. Sorafenib in advanced hepatocellular carcinoma. N Engl J Med. 2008;359(4):378-90.

9. Bruix J, Qin S, Merle P, Granito A, Huang YH, Bodoky G, et al. Regorafenib for patients with hepatocellular carcinoma who progressed on sorafenib treatment (RESORCE): a randomised, double-blind, placebo-controlled, phase 3 trial. Lancet. 2017;389(10064):56-66.

10. US Food and Drug Administration. Regorafenib. Available at: https://www.fda.gov/drugs/informationondrugs/approveddrugs/ ucm555548.htm. Accessed February 28, 2018.

11. Bruix J, Tak WY, Gasbarrini A, Santoro A, Colombo M, Lim HY, et al. Regorafenib as second-line therapy for intermediate or advanced hepatocellular carcinoma: multicentre, open-label, phase II safety study. Eur J Cancer. 2013;49(16):3412-9.

12. US Food and Drug Administration. Nivolumab. Available at: https://www.fda.gov/Drugs/InformationOnDrugs/ApprovedDrugs/ ucm577166.htm. Accessed February 28, 2018.

13. Kudo M, Finn RS, Qin S, Han KH, Ikeda K, Piscaglia F, et al. Lenvatinib versus sorafenib in first-line treatment of patients with unresectable hepatocellular carcinoma: a randomised phase 3 noninferiority trial. Lancet. 2018;391(10126):1163-73.

14. US Food and Drug Administration. Lenvatinib. Available at: https://www.fda.gov/drugs/informationondrugs/approveddrugs/ ucm617185.htm. Accessed October 31, 2018.

15. US Food and Drug Administration. Pembrolizumab. Available at: https://www.fda.gov/Drugs/InformationOnDrugs/ApprovedDrugs/ ucm625705.htm. Accessed February 22, 2019. 
16. US Food and Drug Administration. Cabozantinib Available at: https://www.fda.gov/Drugs/InformationOnDrugs/ApprovedDrugs/ ucm629512.htm. Accessed February 22, 2019.

17. National Comprehensive Cancer Network. Hepatobillary cancer (version 1) 2019. Available at: https://www.nccn.org/ professionals/physician_gls/pdf/hepatobiliary.pdf. Accessed February 22, 2019.

18. Lang K, Danchenko N, Gondek K, Shah S, Thompson D. The burden of illness associated with hepatocellular carcinoma in the United States. J Hepatol. 2009;50(1):89-99.

19. Warren JL, Klabunde CN, Schrag D, Bach PB, Riley GF. Overview of the SEER-Medicare data: content, research applications, and generalizability to the United States elderly population. Med Care. 2002;40(8 suppl):IV-3-18.

20. Tapper EB, Catana AM, Sethi N, Mansuri D, Sethi S, Vong A, et al. Direct costs of care for hepatocellular carcinoma in patients with hepatitis C cirrhosis. Cancer. 2016;122(6):852-8.

21. Shaya FT, Breunig IM, Seal B, Mullins CD, Chirikov VV, Hanna N. Comparative and cost effectiveness of treatment modalities for hepatocellular carcinoma in SEER-Medicare. Pharmacoeconomics. 2014;32(1):63-74.

22. United States Department of Labor. Consumer Price Index details report tables 2015 annual average Available at: https://www.bls. gov/cpi/tables.htm. Accessed July 1, 2018.

23. Parikh ND, Marshall VD, Singal AG, Nathan H, Lok AS, Balkrishnan R, et al. Survival and cost-effectiveness of sorafenib therapy in advanced hepatocellular carcinoma: an analysis of the SEER-Medicare database. Hepatology. 2017;65(1):122-33.

24. Chandra A, Shafrin J, Dhawan R. Utility of cancer value frameworks for patients, payers, and physicians. JAMA. 2016;315(19): 2069-70.

25. Porter ME. A strategy for health care reform-toward a value-based system. N Engl J Med. 2009;361(2):109-12.

26. El-Khoueiry AB, Sangro B, Yau T, Crocenzi TS, Kudo M, Hsu C, et al. Nivolumab in patients with advanced hepatocellular carcinoma (CheckMate 040): an open-label, non-comparative, phase 1/2 dose escalation and expansion trial. Lancet. 2017;389(10088): 2492-502.

27. Brogan AP, DeMuro C, Barrett AM, D'Alessio D, Bal V, Hogue SL. Payer perspectives on patient-reported outcomes in health care decision making: oncology examples. J Manag Care Spec Pharm. 2017;23(2):125-34.

28. Tykodi SS, Schadendorf D, Cella D, Reck M, Harrington K, Wagner S, et al. Patient-reported outcomes with nivolumab in advanced solid cancers. Cancer Treat Rev. 2018;70:75-87.

Publisher's Note Springer Nature remains neutral with regard to jurisdictional claims in published maps and institutional affiliations. 